\title{
Archipel
}

ARCHIPEL Études interdisciplinaires sur le monde insulindien

$101 \mid 2021$

Varia

\section{Archipel illustré - et autres sujets mineurs}

Archipel illustrated - and other minor subjects

Jérôme Samuel

\section{(2) OpenEdition}

Journals

Édition électronique

URL : https://journals.openedition.org/archipel/2425

DOI : $10.4000 /$ archipel.2425

ISSN : 2104-3655

\section{Éditeur}

Association Archipel

Édition imprimée

Date de publication : 30 juin 2021

Pagination : 35-56

ISBN : 978-2-910513-85-6

ISSN : 0044-8613

\section{Référence électronique}

Jérôme Samuel, «Archipel illustré - et autres sujets mineurs », Archipel [En ligne], 101 | 2021, mis en ligne le 30 juin 2021, consulté le 02 juillet 2021. URL : http://journals.openedition.org/archipel/2425 ; DOI : https://doi.org/10.4000/archipel.2425 
JÉROMME SAMUEL*

\section{Archipel illustré - et autres sujets mineurs}

Il faut remonter aux origines d'Archipel pour comprendre la place remarquable que l'illustration y a toujours tenu, remarquable parce que rare dans les revues de sciences humaines et sociales, mais aussi parce qu'inscrite dans l'histoire d'Archipel. Je voudrais revenir brièvement sur quelquesunes des premières orientations de la revue qui me paraissent éclairer cette caractéristique, avant d'en présenter différents aspects.

\section{Une revue pour tous?}

Au-delà du tropisme de ses fondateurs - goût de P. Labrousse pour les beaux-arts et la littérature de voyage, intérêt de D. Lombard pour la cartographie et l'illustration en général, appétence de Chr. Pelras pour le croquis ethnographique - la place accordée à l'image paraît répondre à un choix éditorial implicite, lié aux orientations premières d'Archipel qui, à sa création en 1971, n'est pas pensée comme une revue scientifique classique, en termes de contenu comme de lectorat :

Archipel se propose de publier des chroniques, des études et surtout des documents et des dossiers concernant essentiellement le Monde insulindien. (...) [Archipel veut être] une publication périodique, informant de façon systématique sur l'histoire et l'actualité [du monde insulindien] (...) [elle veut constituer] avant tout un trait d'union (...) entre les

* CASE, INALCO 
diverses méthodes qui (...) abordent [le monde insulindien] en ordre dispersé et surtout entre "spécialistes" et "non-spécialistes" qui s'affrontent en une querelle inféconde. (An., $1971, \mathrm{n}^{\circ} 1$, p. 3 ; souligné par nous) ${ }^{1}$.

En effet, l'idée d'Archipel n'est pas seulement née de jeunes indonésianistes dans les débuts de leur carrière, mais aussi d'échanges à Jakarta au tournant des années 1970, entre cette poignée de chercheurs et un cercle local d'expatriés - cadres des industries pétrolière, aéronautique, de la diplomatie, etc. - intéressés par le monde insulindien, désireux de retirer de leur séjour en Indonésie autre chose que des " satisfactions esthétiques » personnelles et d'y trouver une « rencontre véritable », pour reprendre les mots de Chr. Pelras ( $c f$. note 1 ci-dessous). Cette proximité explique une part, certes jamais dominante mais notable, de la production éditoriale de la revue pendant les quinze premières années de son existence : articles étrangers aux sciences humaines mais relevant du domaine de compétence de ces expatriés (économie, commerce, industrie ${ }^{2}$ ), ainsi que ces rubriques grand public qui firent un des charmes de la revue. On pense ici aux « Guides Archipel », aux « Pages d'exotisme » et aux chroniques annuelles d'Indonésie - ces dernières continuèrent jusqu'en $2011^{3}$-, et dont les fondateurs de la revue furent aussi les plus assidus rédacteurs (voir en bibliographie). La présence de « petits » sujets - entendre : encore peu traités par les sciences sociales - trouve peut-être son origine dans ce même milieu, comme ils témoignent d'une approche « naïve » - comprendre : sans préjugés - de champs nouveaux qui ne demandaient qu'à être explorés, et dont l'ancrage dans l'aire culturelle insulindienne ajoutait encore à l'intérêt : cinéma (numéro spécial « Le Cinéma indonésien »1973, $\mathrm{n}^{\circ} 5$ ), bande dessinée ${ }^{4}$, par exemple, mais aussi littérature sino-malaise (voir 1'article de Cl. Salmon dans le présent numéro).

Il faut ajouter à ce curieux entre-deux éditorial, le fait qu'à quelques exceptions près la recherche et l'université française n'avaient guère produit de spécialistes du monde insulindien jusque-là, du moins comparativement

1. La même idée revient quelques années plus tard sous la plume de Chr. Pelras, qui conclut : «Une des missions d'Archipel est d'aider à une telle rencontre. » (1975, $\left.{ }^{\circ} 10: 50\right)$. P. Labrousse nuancera cette candide opposition binaire, dans une de ses « Pages d'exotisme », consacrée aux « bateleurs au verbe haut, à la faconde intarissable, mystificateurs exploitant sans vergogne l'ignorance du public sur l'Indonésie. » (1976, n¹1, p. 107).

2. Voir fig. 3 infra et l'auteur de l'article dont elle est extraite.

3. Fr. Raillon en fut longtemps l'auteur (1990, n³9-2011, n81), sous l'intitulé « Chronique du temps présent $\gg$.

4. La bande dessinée indonésienne a fait l'objet de la thèse de M. Bonneff (« Les bandes dessinées indonésiennes : une mythologie en images ", Paris : EPHE, 1972, publiée en 1976), d'où les deux articles qu'il a livrés en $1972\left(\mathrm{n}^{\circ} 4\right)$ et $1973\left(\mathrm{n}^{\circ} 5\right)$, ainsi qu'un compte rendu de lecture de l'album d'Hergé Vol 714 pour Sydney dans le premier numéro de la revue. Le même compte rendu est suivi d'un autre consacré à un livre pour enfants : D. Darbois, Rikka la petite Balinaise (Paris : Nathan, $19561^{\mathrm{e}}$ éd.). 
à leurs homologues néerlandais ou anglo-saxons, alors que la dimension nationale (française) ou bilatérale (franco-indonésienne) de la revue n'était pas moins clairement affirmée, comme le rappelait Chr. Pelras en 1998 :

Il s'agissait d'offrir enfin un moyen d'expression aux chercheurs français en sciences humaines et sociales qui, en nombre croissant, commençaient à s'intéresser à l'Indonésie et aux régions voisines, et qui manquaient jusque-là d'une publication spécialisée où faire paraître, en français, les résultats de leurs recherches. On souhaitait aussi en élargir les perspectives à l'ensemble du "monde malais" et offrir la possibilité à des chercheurs de la région de faire connaître, par cet intermédiaire, leurs travaux (dans leur langue nationale ou en traduction) au public international. (Chr. Pelras, $1998: 8$; souligné par nous) ${ }^{5}$

Tout cela a dû laisser sceptiques les concurrents de la revue, à commencer par les mieux établis (BEFEO, BKI, JMBRAS) et même les plus récents (Indonesia, Indonesia Circle), de l'époque.

Les premières orientations " généralistes 》d'Archipel, donc le souci de produire un discours scientifique accessible à d'autres que les spécialistes des domaines abordés, la volonté de privilégier l'expression en langue française s'effacent, très rapidement pour certaines, plus progressivement pour d'autres. Dès son numéro deux (1972), la revue s'ouvre à des auteurs étrangers, mais il est à souligner que ces contributeurs seront d'abord indonésiens (Ajip Rosidi, Wing Kardjo) puis malaisiens (Syed M. Naguib al-Attas, 1972, $\mathrm{n}^{\circ} 4$ ) en traduction française et, après seulement, néerlandais et britanniques (Chr. Hooykaas, P. Voorhoeve, R. Jones, 1973, nº6)

Cette évolution reflète moins une qualité scientifique accrue des contenus - cette exigence a toujours été celle de la rédaction -, que l'insertion de la revue dans un environnement scientifique international et, plus tard, l'incitation d'acteurs institutionnels qui attendent des revues qu'elles répondent à un ensemble de normes scientifiques, éditoriales (présence de titres courants à partir du $\mathrm{n}^{\circ} 49,1995$ ), linguistiques (place grandissante de l'anglais), auxquelles Archipel se pliera pour l'essentiel. C'est donc à la fois la rançon du succès et la nécessité de la survie : accès aux parrainages institutionnels et aux moyens financiers, mais aussi techniques et humains, indispensables à la production d'objets de qualité.

\section{La place de l'image}

À une époque pas si lointaine où l'image était plus rare qu'aujourd'hui, surtout pour cet angle mort de l'Asie en France que constituait encore le monde insulindien, il était nécessaire de lui faire une place et cette nécessité

5. Les articles en indonésien ou en malaisien n'ont jamais été exclus mais, de fait, jamais très nombreux non plus. Les premiers numéros d'Archipel incluaient un résumé des articles (ikhtisar) en indonésien.

6. Russel Jones livre à cette occasion le premier article en anglais de la revue. 
a paru d'autant plus évidente, que la revue voulait s'adresser à ce lectorat élargi, en partie étranger au monde de la recherche en sciences humaines et au discours savant, qui se nourrit plus volontiers d'illustrations. C'est aussi en cela qu'Archipel se distingue, car si toutes les revues de sciences humaines font une place à l'illustration, souvent limitée à divers graphiques et schémas, parfois incluant photos et dessins, en dehors des revues d'archéologie, d'histoire de l'art ou d'arts plastiques, il n'est pas fréquent qu'elles y accordent autant d'importance que l'a fait Archipel, et la pluridisciplinarité revendiquée et réelle de la revue n'y est pas pour rien. En revanche, nulle réflexion théorique ou méthodologique sur la place et la fonction de l'image dans ce type de discours et dans ces disciplines; il est vrai qu'au début des années 1970, cette approche était encore embryonnaire.

\section{Abondance d'images}

Dès le premier numéro de la revue Archipel, il apparut donc clairement que l'image pourrait y tenir une place importante, du moins au regard des usages : 20 illustrations dont 12 pages hors texte pour un volume de 247 pages. Après quoi et pour les 50 années d'existence de la revue, les statistiques confirment l'impression dégagée par la simple consultation des volumes : généralement importantes en proportion, les images sont aussi présentes en nombre croissant malgré les fluctuations ${ }^{7}$. Le graphique 1 en donne la variation par numéro (histogramme bleu) et avec une moyenne lissée sur cinq ans (courbe orangée), où l'on voit que cette moyenne a presque doublé sur l'ensemble de la période :

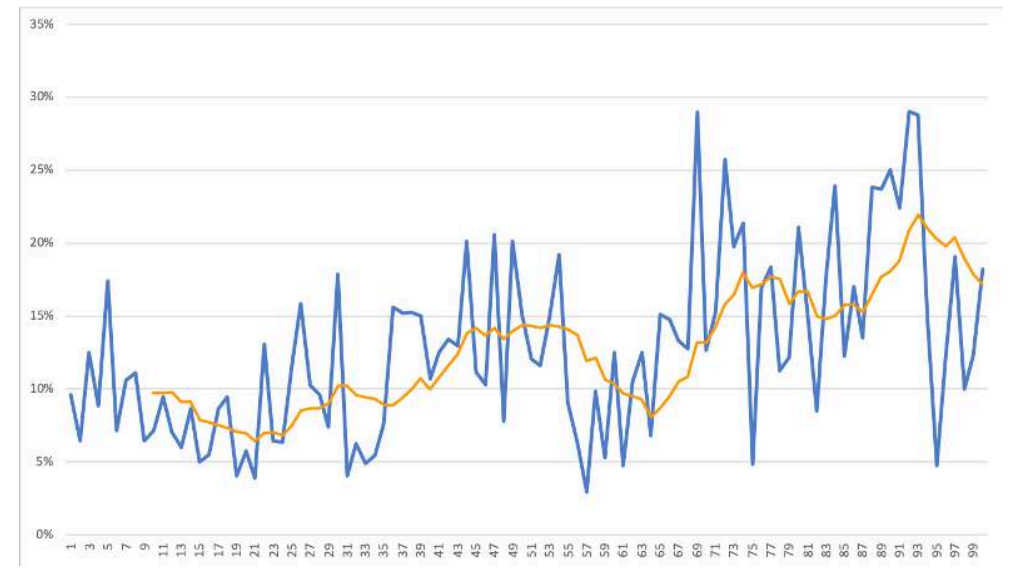

Graphique 1 - Pourcentage de pages illustrées dans chaque numéro.

Bleu : valeurs par numéro - Orangé : moyenne mobile sur cinq ans.

7. Ce dont ne rend pas compte la consultation en ligne sur le site $<$ persee.fr $>$, où manquent de très nombreuses illustrations. 
Par ailleurs, pour la totalité de la période la proportion moyenne de pages illustrées par numéro s'établit à $12,68 \%$. Aucun volume n'est vide d'illustrations, un gros tiers des numéros a moins de $10 \%$ de pages illustrées, la moitié (49 exactement) en a entre $10 \%$ et $20 \%$, et près de 15 volumes (14 exactement) en ont $20 \%$ ou plus, proportion considérable. Cette présence est donc régulière. Elle progresse aussi dans le temps : si l'inflexion est donnée dès le numéro 5 (avec 17,4\% de pages illustrées portées, il est vrai, par une thématique qui s'y prête : le cinéma), l'effort est nettement accentué à partir du n $\mathrm{n}^{\circ} 4(20 \%)$ et le maximum atteint avec le $\mathrm{n}^{\circ} 69$, premier de deux volumes consacrés à la peinture à Java (29\%). Néanmoins, on observe une forte irrégularité entre les numéros, ce qu'explique l'absence de politique éditoriale en la matière.

Quant aux modes d'insertion des illustrations, on relève une double évolution. Tout d'abord la présence de plus en plus irrégulière des illustrations pleine page (graphique 2, courbe jaune) : longtemps en nombre plus ou moins constant et insérées sous forme de cahiers de quatre à huit pages hors texte pour des raisons techniques, elles finissent par se concentrer sur certains articles, dont la rubrique «Images » constituée en cahier. Corollairement, les progrès de la mise en page et l'adoption d'un format de revue légèrement plus grand (1991, $\left.\mathrm{n}^{\circ} 41\right)$ facilitent la disposition d'illustrations dans le texte, dont le nombre finit par s'envoler au cours des années 2010, en particulier dans les articles d'épigraphie et d'archéologie (graphique 2, courbe grise).

La couleur, enfin, a longtemps été un luxe peu accessible réservé aux couvertures, sobrement bicolores sur fond blanc. La quadrichromie s'affiche timidement en couverture du nº51 (1996) et se glisse dans le même volume avec un premier cahier central en quadrichromie (fig. 7), entraînant un surcoût de fabrication âprement discuté ; elle revient l'année suivante dans le ${ }^{\circ} 54$ qui avait fait l'objet d'un effort tout particulier («Destins croisés entre l'Insulinde et la France », 1997), se banalise un peu plus tard (2001) avant de devenir la règle en à partir de 2004. Elle est aujourd'hui omniprésente et les nouveaux modes de diffusion (consultation sur écran, accessibilité de l'impression couleur domestique ou à la demande) font que la question n'est plus de savoir pourquoi la revue Archipel offre autant de pages illustrées et de pages en couleurs, mais plutôt pourquoi les autres revues couvrant les mêmes champs disciplinaires ou aréaux en incluent si peu...

\section{Nature des illustrations}

Les illustrations sont de toutes natures, mais le dessin et, surtout, la photographie dominent : de lieux, de bâtiments, d'objets - d'images : peintures, gravures, dessins -, mais aussi de personnes, et bien entendu de documents textuels sur différents types de supports. On opposera ici ces derniers (inscriptions, manuscrits) aux autres. Leur reproduction s'impose d'autant plus que les usages de la discipline exigent de mettre à la disposition 


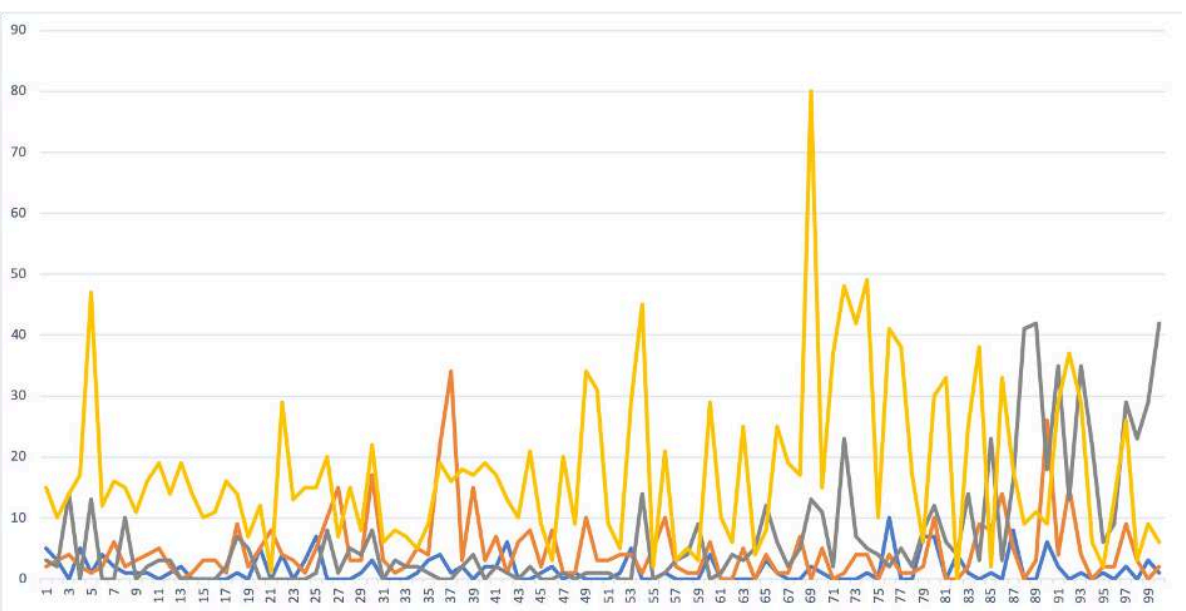

Graphique 2 - Nombre de pages illustrées dans chaque numéro. Répartition par types d'illustrations et modes d'insertion.

Bleu : graphiques, schémas, généalogie - Orangé : cartes - Gris : dessins, photos, etc., dans le texte-Jaune : idem hors texte.

des lecteurs - l'infime minorité de lecteurs capable de le lire - le texte sous sa forme originale avant d'en proposer une lecture, et que la longueur du document en rend possible la reproduction. Cela concerne essentiellement l'épigraphie, à commencer par l'épigraphie funéraire musulmane, la mieux représentée, et dans une moindre mesure la philologie ; mais les articles à vocation d'analyse littéraire ou fondés sur des documents imprimés sont aussi consommateurs d'illustrations de nature textuelle.

Les autres types de documents tiennent une place marginale : graphiques et schémas (ceux-là ont mal résisté au temps), généalogies ; cartes et plans (élégamment tracés à la main dans les premières années de la revue). Pour les cartes, il faut d'ailleurs distinguer deux cas différents, selon qu'elles viennent à l'appui d'un discours de nature géographique ou historique sur l'espace considéré ou que le discours porte sur le document présenté (cartes anciennes) et il s'agit alors d'images; dans une revue historique, il est normal que les deux se présentent.

\section{La rubrique "Images " ou l'échec d'un discours sans texte}

Apparue dans le $\mathrm{n}^{\circ} 42$ (1991), juste après que la revue eut agrandi son format, la rubrique «Images » avait d'autres ambitions que la simple illustration d'articles ou de dossiers, mais son introduction n'est pas à prendre pour une tentative de cantonnement de l'image ; elle répond à la volonté de proposer un autre type d'articles ou de discours, sans renoncer nécessairement à sa scientificité. En effet, initialement cette rubrique avait été destinée à 
« explorer les fonds d'archives photographiques relatifs à l'Indonésie, pour mieux attirer l'attention sur des documents souvent mal connus et rarement exploités par les historiens. » (Labrousse 1991, n42, p. 121). Malgré cette déclaration liminaire, la rubrique et ses auteurs ne puisèrent pas aux archives

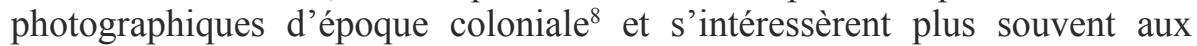
gravures et dessins d'une période plus large (XVII ${ }^{\mathrm{e}} \mathrm{XIX}^{\mathrm{e}}$ siècles) ou, par la photo, à la période contemporaine (voir bibliographie).

La seconde ambition de cette rubrique - «le commentaire est volontairement bref, afin de laisser la place aux images » (Labrousse, ibid.) - a tourné à l'échec. Certes, la première livraison respecta parfaitement ce cahier des charges, mais ces quelques photographies issues de la documentation de la Fondation Idayu (Jakarta) et couvrant la période de transition entre le 30 septembre 1965 et les premiers mois de l'année suivante (voir infra fig. 4), traitaient d'événements relativement récents et dont on pouvait juger qu'ils ne nécessitaient pas de remise en contexte. Ce choix, pourtant, tournait le dos à l'intention originelle d'Archipel de s'adresser aussi bien à un lectorat néophyte et reposait sur l'illusion d'images porteuses de leur propre discours.

D'ailleurs, dès le numéro suivant, des légendes plus ou moins détaillées vinrent s'ajouter à des introductions parfois très longues (Lombard, 1992, $\mathrm{n}^{\circ} 43$ ). Dès lors, à quelques exceptions près cette rubrique proposa de véritables articles prenant appui sur de petits corpus d'images, sans se distinguer d'articles de recherche très illustrés ou dont les images présentées constituaient l'objet même de l'étude, en particulier les articles d'histoire de l'art.

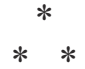

En guise de conclusion, voici treize photos extraites de différents numéros d'Archipel. Elles accompagnent cette note plus qu'elles ne l'illustrent : leur choix, purement subjectif, repose sur des critères émotionnels ou esthétiques. Cette démarche et la sélection qu'elle commande nous a paru préférable à un catalogue reproduisant un à un les différents types d'images offerts par la revue $^{9}$. Ces photos, prises par des contributeurs de la revue à trois exceptions près (fig. 3,9 et 10), rappellent aussi qu'historiens, anthropologues ou autres, tous sont aussi leur propre photographe et chasseur d'images.

8. À l'exception de Laffan, 2003, n65.

9. Les références complètes des articles dont ces illustrations sont extraites se trouvent en bibliographie. 


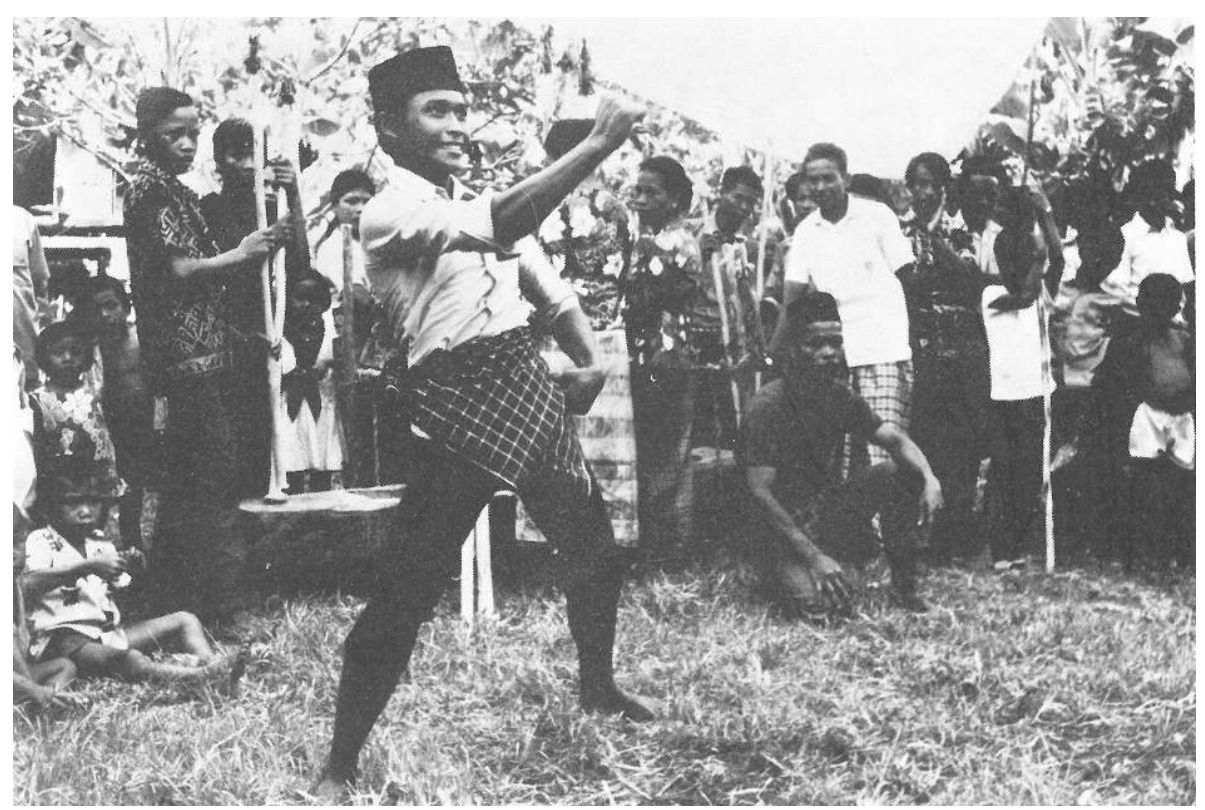

Fig. 1 -. Chr. Pelras, 1975. Photos auteur, s.d.

Légende - « Fête de la moisson en pays bugis : au rythme des pilons à riz, les jeunes gens viennent tour à tour démontrer leur maitrise des arts martiaux (silat). » (pl. h.t., face p. 48)

Le $\mathrm{n}^{\circ} 10$ de la revue fut le premier et l'un des rares entièrement consacrés à une région d'Indonésie, ici la province de Célèbes sud. Les illustrations n'étaient pas encore nécessairement placées à proximité des textes auxquels elles se rapportaient. Les photos que voici accompagnaient un guide touristique de la province, conçu par un anthropologue pour un public attendu d'amateurs éclairés ou de simples touristes - les deux groupes se confondaient plus ou moins en 1975 (voir supra).

Ces deux photos (fig. 1 et 2) ne se répondent pas comme elle semblent le faire de prime abord.

La première illustration évoque un univers festif, animé, lieu d'une violence ritualisée, de celles qui suscitent la connivence et le sourire : à l'arrogance virile et joyeuse d'un jeune homme, raide, poing serré et tendu, campé sur ses jambes, bassin projeté en avant, répondent les sourires qu'on devine à l'arrière-plan. 


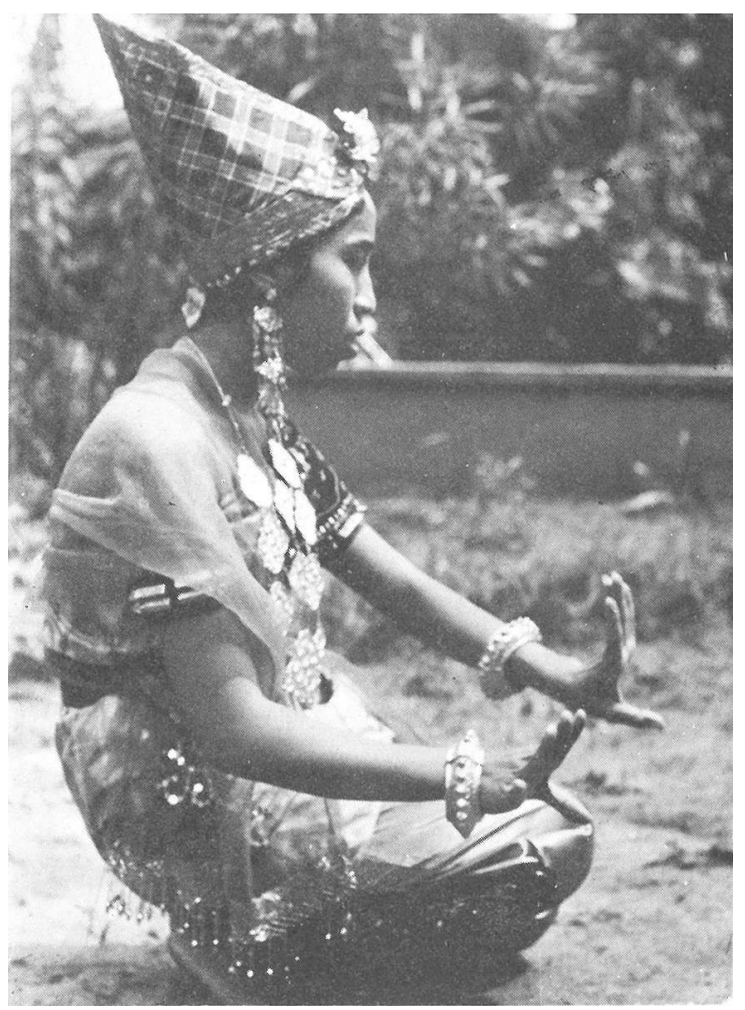

Fig. 2 -. Chr. Pelras, 1975. Photos auteur, s.d.

Légende - « Séngkang : danseuse de séré lolosu. » (pl. h.t., face p. 96)

L'auteur en dit peu sur la seconde illustration : il omet de préciser que la danse [sere] des lolosu [nom des accessoires représentant des coqs s'affrontant l'un l'autre (Hamonic, 2006, p. 9 n. 2] est exécutée par des bissu, prêtres et guérisseurs traditionnels travestis. Le raffinement féminin de ce-tte danseur-se, couverte de bijoux et d'étoffes délicates, tout en courbes et en souplesse, est celui d'un de ces jeunes prêtres. 


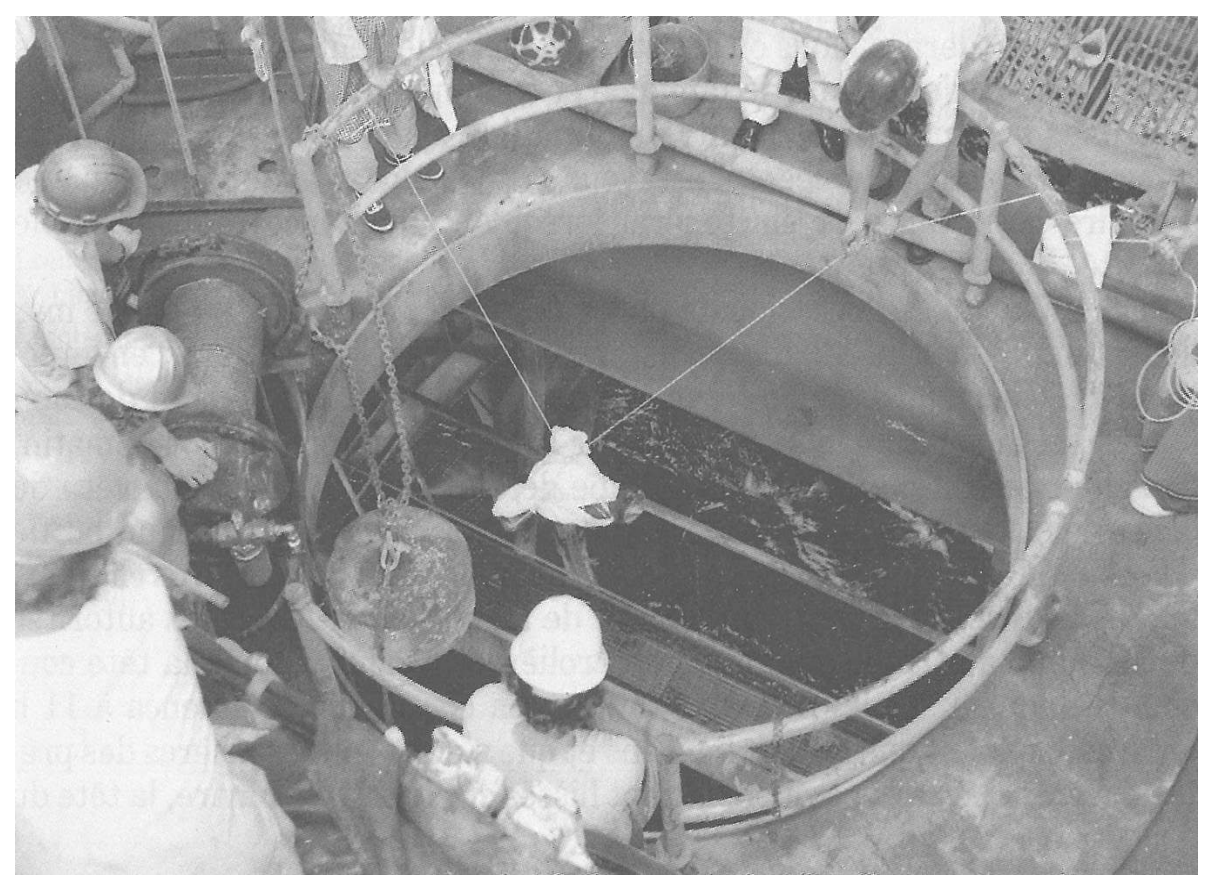

Fig. 3 - A. Francino, 1990. Photos auteur, 1975.

Légende - « Moment culminant de la cérémonie du salamatan [sic] : la tête du buffle va être immergée près de la colonne de forage. » (p. 61)

Le maître-mot de ces deux photos (fig. 3 et 4), sans autre rapport entre elles, pourrait être celui de sacrifice.

Sacrifice d'un buffle et immersion de sa tête enveloppée dans un tissu blanc, sur un forage off shore en mer de Natuna (on devine l'écume des vagues en contrebas de la plateforme). Le contraste entre ce rituel propitiatoire et le contexte industriel dans lequel il prend place intéresse-t-il ses acteurs ou ne germe-t-il que dans l'esprit du lecteur? L'auteur de l'article, géologue de son état et très factuel, se borne à rappeler que « [cette] offrande faite à la mer revêt la même valeur que les offrandes faites autrefois aux vents, pour que les forces naturelles ne s'acharnent pas trop contre la fragilité humaine. Souvent, d'octobre à mars, les tempêtes et les typhons qui se déchaînent dans la mer de Natuna rendent les opérations de forage marin très critiques en raison des tempêtes qui contraignent de suspendre les activités. » (p. 63) 


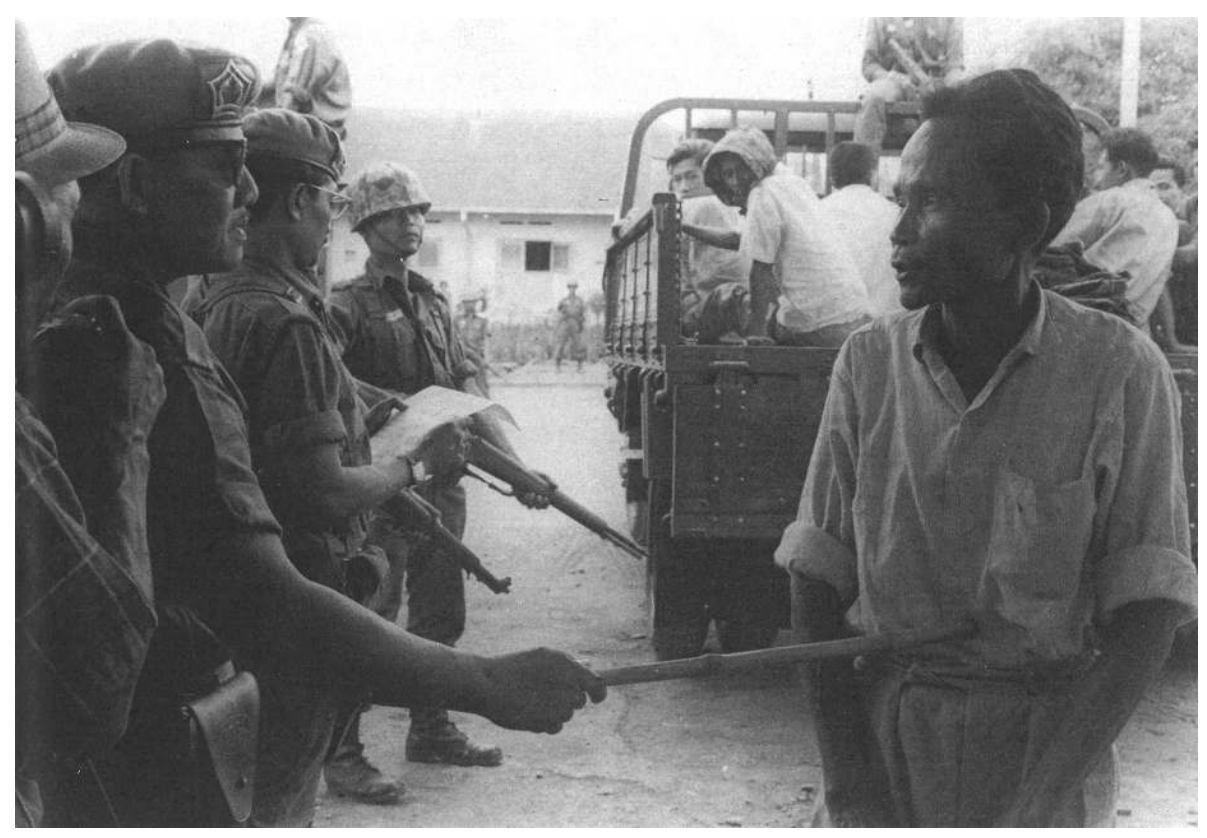

Fig. 4 - P. Labrousse, rubrique « Images », 1991. Photo Fondation Idayu, $1^{\text {er }}$ novembre 1965.

Légende - «Jakarta, $1^{\text {er }}$ novembre 1965. Les premières arrestations des communistes soupçonnés d'être impliqués dans le coup du 30 septembre. » (p. 48)

Sacrifice également, mais sur l'autel de l'anticommunisme, avec la seconde photo. Au-delà de la confrontation des regards, celui terrifié du suspect (peutêtre un simple passant) flottant dans des vêtements mal ajustés, face à celui de l'officier, moue menaçante, masque des lunettes noires (cacher son regard: autre privilège de l'autorité), tout est dans cet avant-bras nu prolongé d'un stick, l'extrémité plantée dans le ventre du suspect et qui l'immobilise. Il y a, dans cette tige de bambou, plus de violence que dans toutes les armes réunies sur cette photo. Peut-être l'homme allait-il s'éloigner, puisqu'il tourne le dos au camion où sont chargés d'autres suspects. Des paroles s'échangent. Un drame se noue entre ces deux hommes, fugitivement ou à jamais : l'un a pouvoir de vie et de mort sur l'autre, et chacun en a pleinement conscience. 


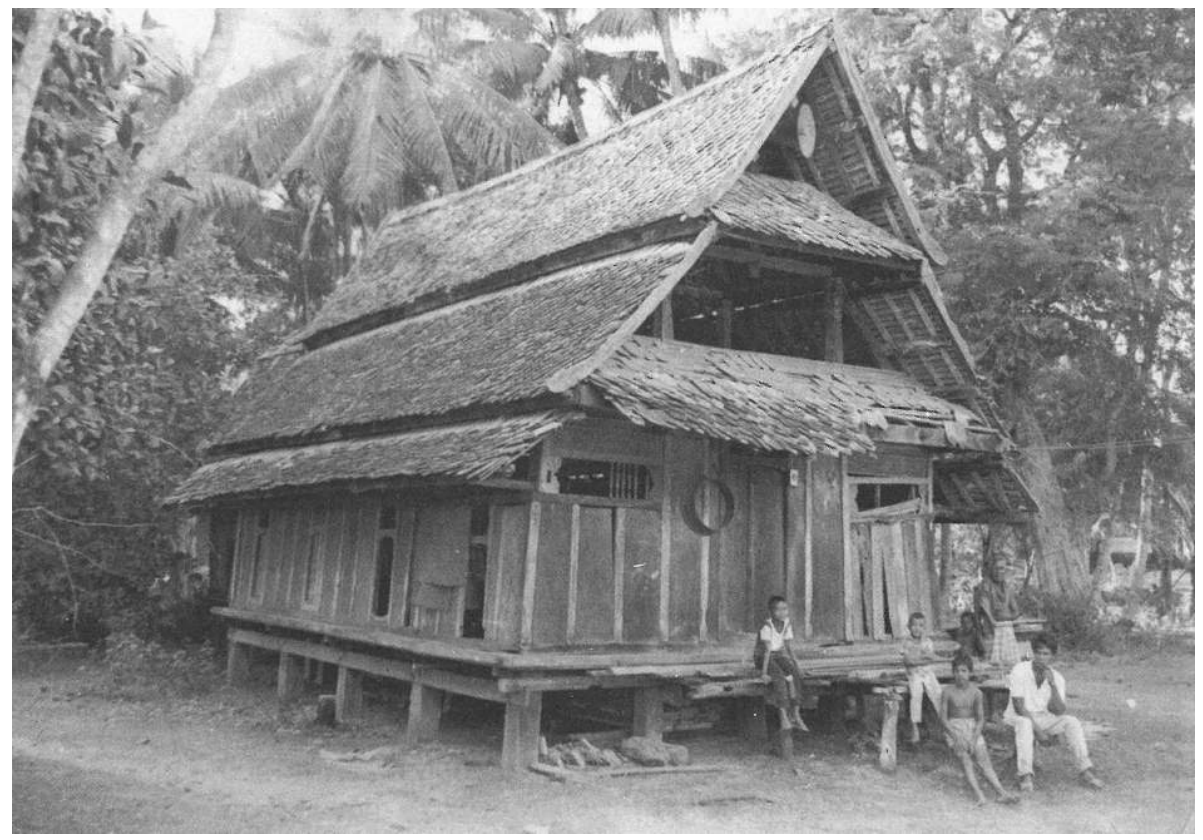

Fig. 5 - W.A.L. Bougas, 1992. Photo auteur, s.d.

Légende - « Surau Aur, Prayer Hall (from the East)» (p. 93)

Deux lieux d'expression de l'islam et de la religiosité dans le monde malais, la mosquée (Patani, fig. 5) et le lieu de pèlerinage (Java, fig. 6). Dans les deux cas et surtout le second, on retiendra que le chercheur n'évolue jamais seul, mais toujours ou presque sous le regard de guides d'un jour, d'autorités locales ou de simples badauds. Ils peuvent donner vie à un site et rappeler qu'il reste fréquenté et honoré, malgré son délabrement, mais ils sont ailleurs l'objet même de l'enquête.

Sur la photo de la mosquée d'Aur, ces gens occupent le lieu dans des attitudes de la vie quotidienne, négligées, à peine posées, par lesquelles ils manifestent qu'ils sont ici dans un espace familier, à la différence du chercheur. 


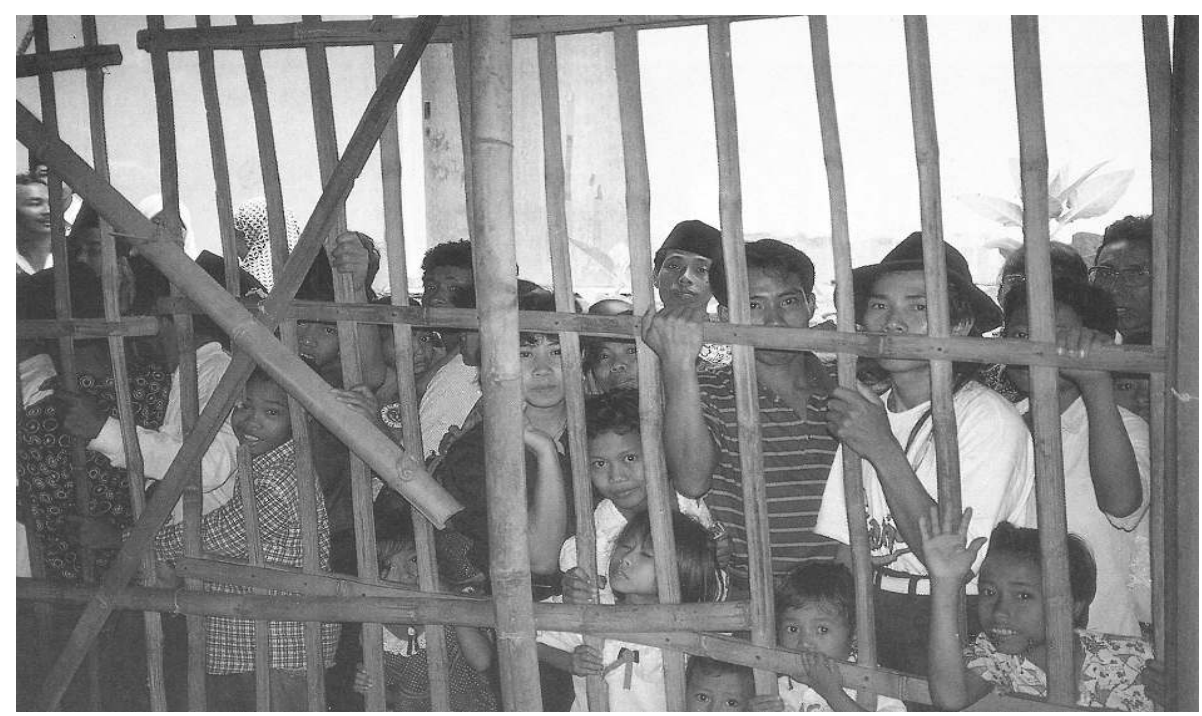

Fig. 6 - Cl. Guillot et H. Chambert-Loir, rubrique « Images », 1993. Photo auteurs, s.d.

Légende - « [Auprès du mausolée], la foule est si dense que, dans l'immense et nouveau pendopo qui recouvre toutes les tombes du complexe, il a fallu mettre en place barrières de bambou et gardes pour la canaliser. » (p. 103)

Au mont Muria la présence de chacun, pèlerins et chercheurs, repose aussi sur des légitimités différentes, mais au fond personne n'est chez soi. Deux mondes s'observent avec une acuité saisissante et la barrière de bambou n'est pas sans susciter un certain malaise. Les chercheurs, ni musulmans ni indonésiens, donc à la légitimité mal reconnue (qu'éprouvent-ils de la barokah du saint ?), ont pu se ménager un passage vers l'intérieur du bâtiment, à la différence des pèlerins qui se pressent encore au dehors; les positions sont donc inversées, au contraire de ce que suggère l'image, pèlerins encore exclus du sanctuaire et chercheurs enfermés à proximité de la tombe du saint.

Enfin, à bien examiner les pèlerins, il paraît difficile de déterminer de quels côtés de la barrière sont le spectacle et les spectateurs, quoi que suggère la légende de la photo. Les enfants, main levée et sourire aux lèvres, semblent se placer côté scène, mais on n'en dira pas autant des adultes. 


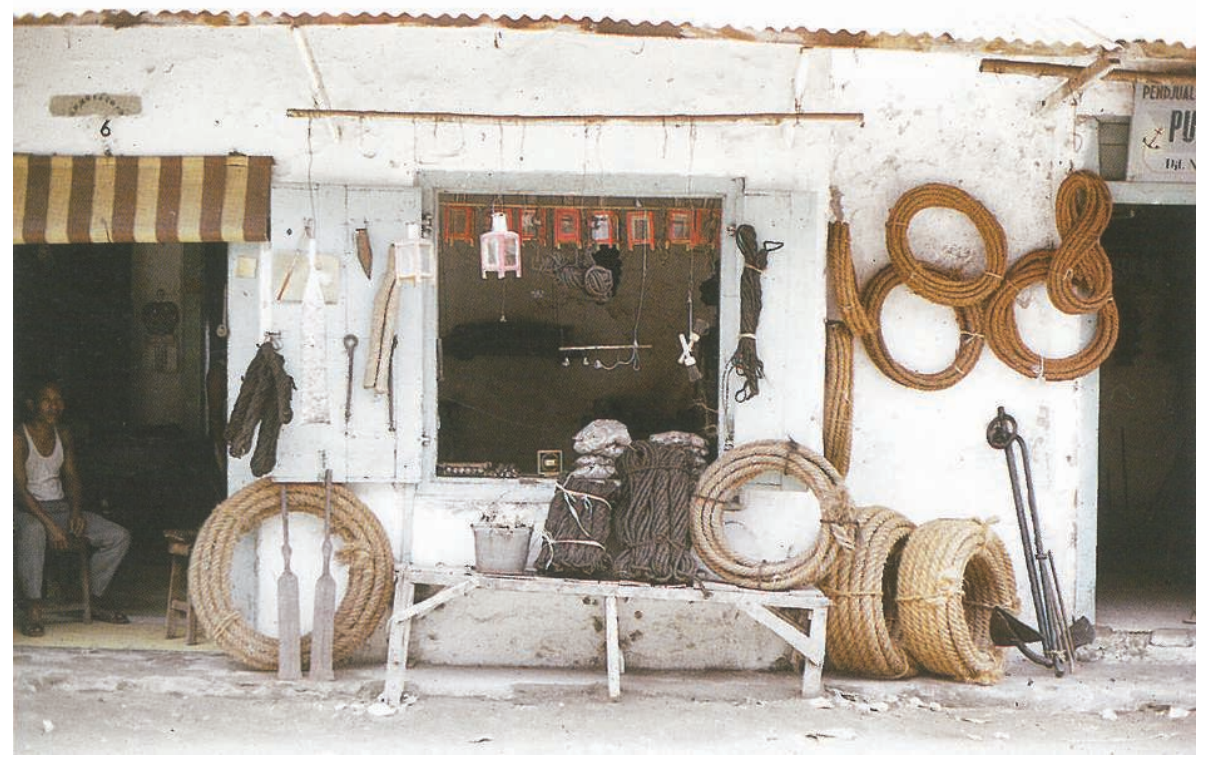

Fig. 7 - P. Piollet, Cl. Salmon et D. Lombard, rubrique « Images », 1996. Photo P. Piollet, 14 février 1974.

Légende - « Penjual alat perahu; boutique d'accessoires pour la navigation à voile : cordages, ancres, fanaux, et voiles (à l'intérieur...) » (p. 102)

Ces deux photos (fig. 7 et 8 ) quasi vides d'hommes donnent à voir un univers disparu ou en voie de l'être, dans une même région, la côte nord de Java.

Le magasin d'articles de mer appartient au monde de la navigation à voile, sur le déclin depuis plusieurs décennies et il a pu faire figure de curiosité déjà lors de la publication de cette photo. La surexposition de la photo s'ajoute à l'absence d'ombre, au négligé du commerçant et rend visuellement sensible la chaleur implacable de la côte javanaise. 


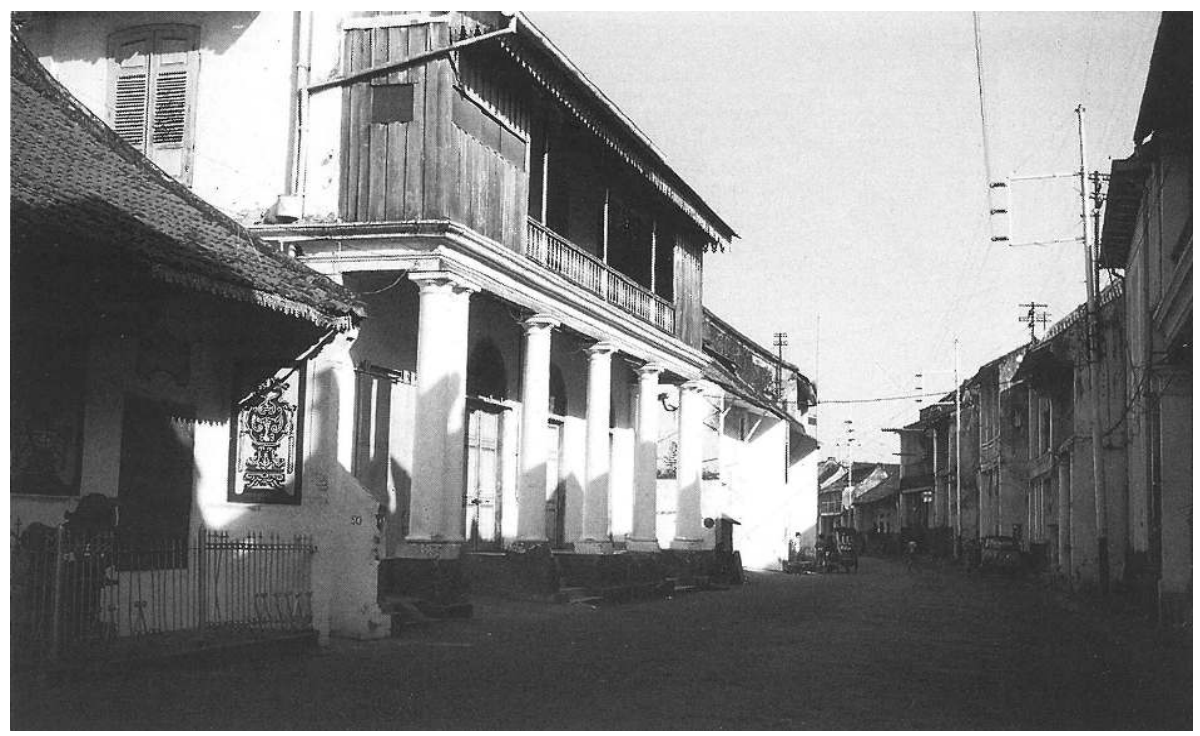

Fig. 8 - Cl. Salmon, 1997. Photo D. Lombard, s.d.

Légende - « Le cœur de l'ancien quartier chinois de Surabaya, actuellement jalan Karet. » (p. 183)

Cette vue de jalan Karet, plongée dans la fraîcheur renaissante d'une fin de journée appartient aussi au passé : ces bâtisses d'architecture coloniale, quand elles subsistent, n'alignent plus que leurs façades tavelées de champignons ou recouvertes de panneaux criards devant un flot ininterrompu de véhicules. 


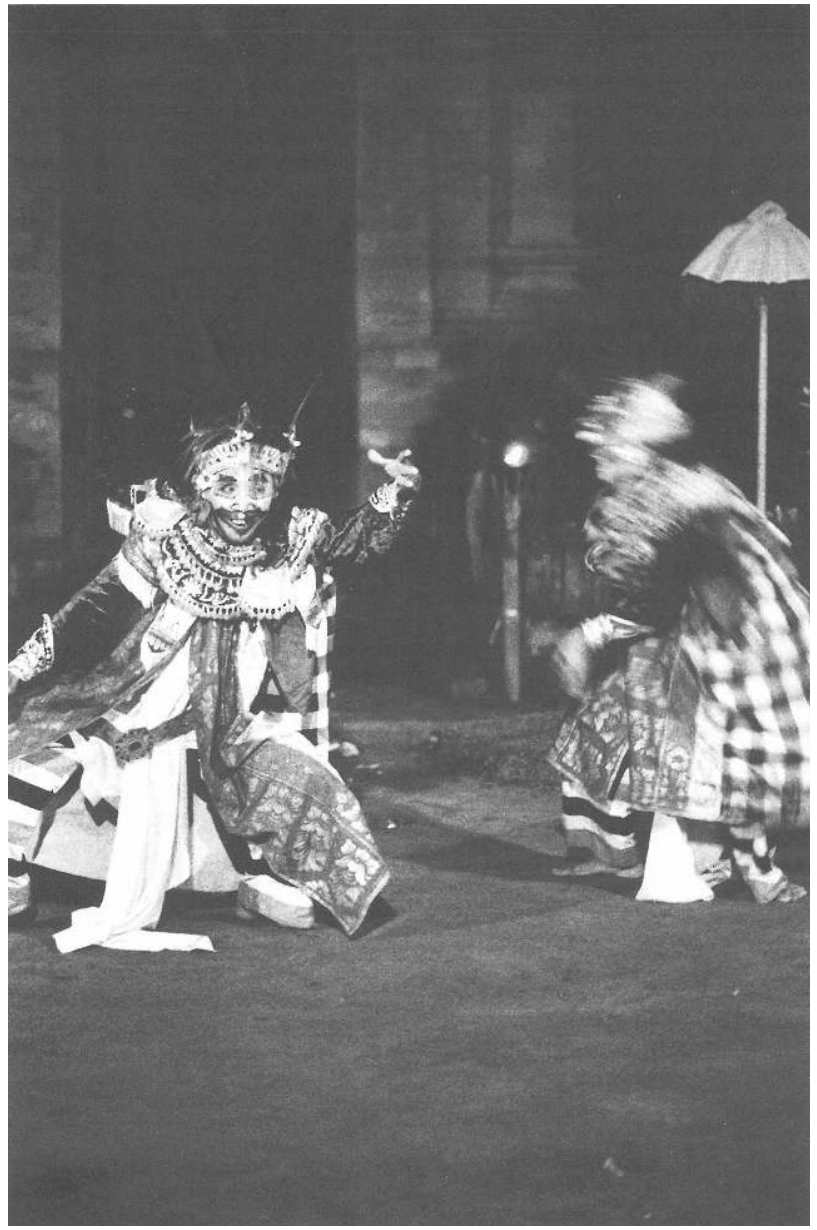

Fig. 9 - M. Picard, rubrique « Images », 1998. Photo K. Helmi, s.d.

Légende - « Tumenggung et Demang (deux grands du royaume accompagnant le souverain), bourg de Batuan ». (p. 189)

Les articles de la rubrique Images aussi bien que les articles de recherche ont souvent inséré des œuvres d'art prises en tant que telles (peintures, gravures), mais très occasionnellement des photos à vocation esthétique. Le flou qui baigne l'un de ces clichés et traverse l'autre semble également les rapprocher, mais si l'un (fig. 9) évoque le mouvement, celui du gambuh balinais, l'autre (fig. 10) rend un monde vacillant, celui des premières années de la Reformasi et de ces vagabonds, fous vrais ou faux comme le suggère l'auteur de l'article, et qu'il n'est pas rare de croiser en ville, évoluant à demi-nus par tous les temps - ici pluie et froid. 


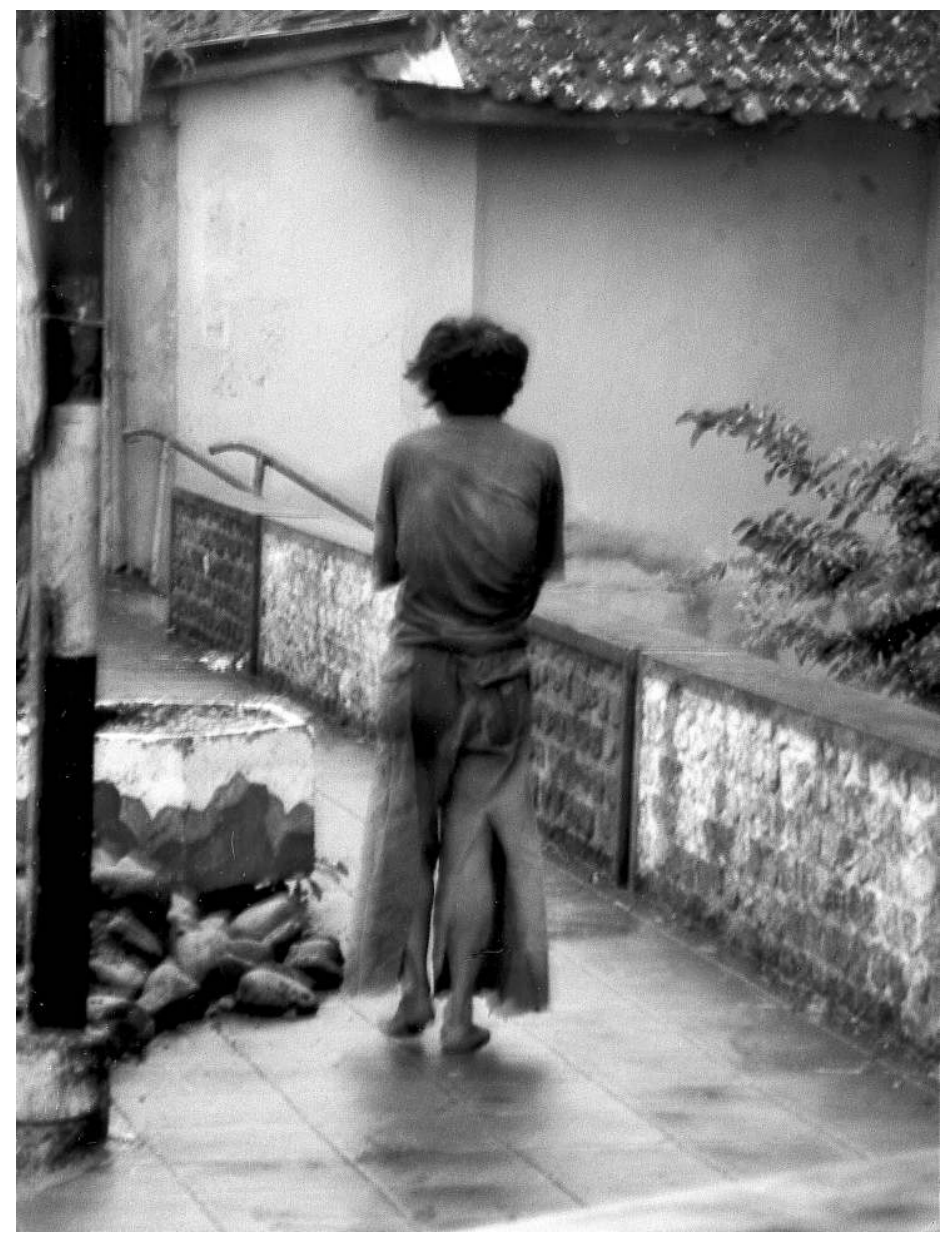

Fig. 10 - J. Siegel, rubrique « Images », 2002. Photo Y.T. Yovovski, s.d.

Légende - «A mad man in Banyuwangi. Members of the Nahdatlul Ulama leadership told us that during the killings, the population of such people drastically increased. The newcomers however had accents from other parts of Java. Members of masked gangs were arrested who were really criminals or soldiers, we were told. But madmen were released in their place. Alternatively, they said that madmen would be chased, caught and turn out to be soldiers. » (p. 180) 


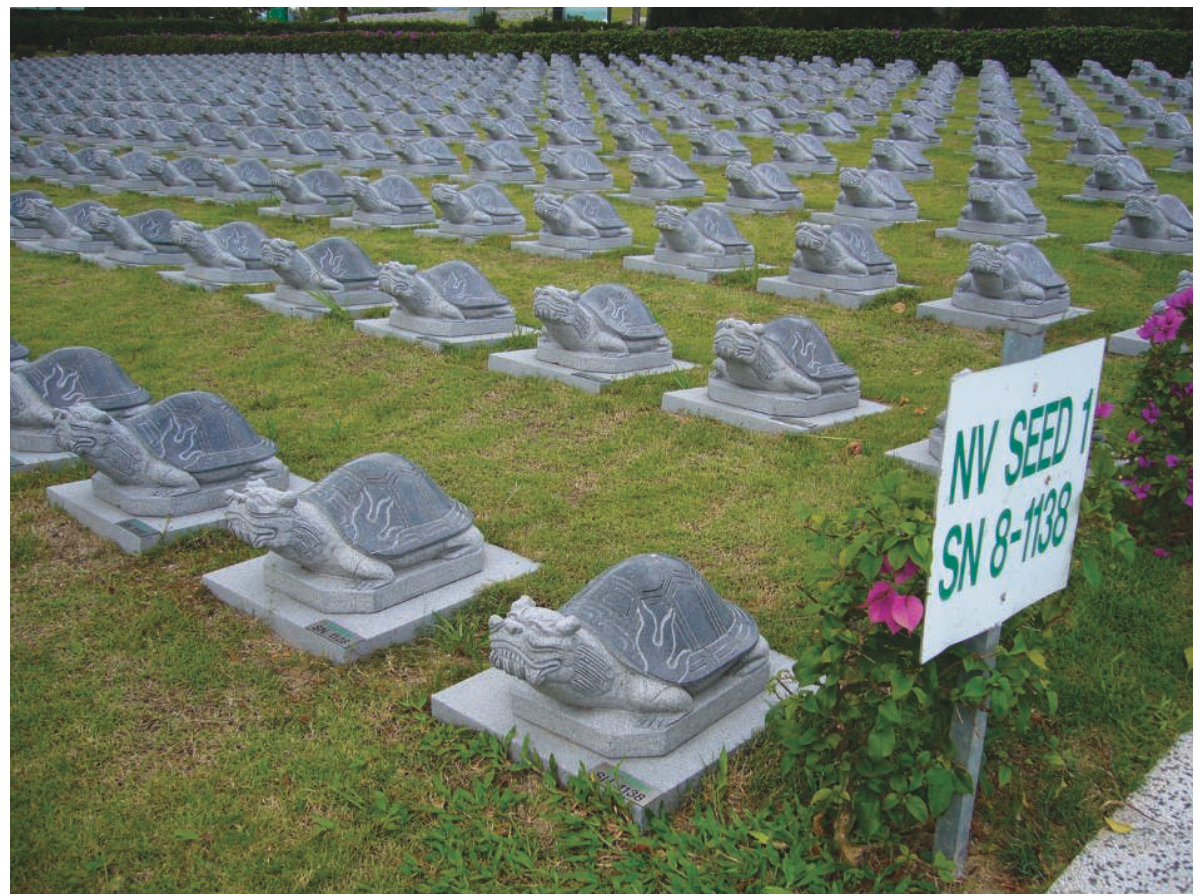

Fig. 11 - Cl. Salmon, 2016. Photo Cl. Salmon, s.d.

Légende - « Special section for shengji or 'foundation of destiny', Nirvana Memorial Garden [Semenyih, Selangor, Malaysia]. » (p. 210)

Tortues d'immortalité dans un parc funéraire de Malaisie et palanquins des morts erong en pays Toraja. Aux alignements impeccables de ces tortues de marbre, toutes identiques et scellées en terre à jamais, répondent ces sépultures d'un moment (fig. 12), longuement, finement ouvragées dans d'infinies variations qui font de chacune un objet unique, comme celle ou celui qui l'habitera le temps d'un ultime transport, les morts rejoignant ensuite leur place assignée à flanc de montagne - et les palanquins jetés au rebut. 


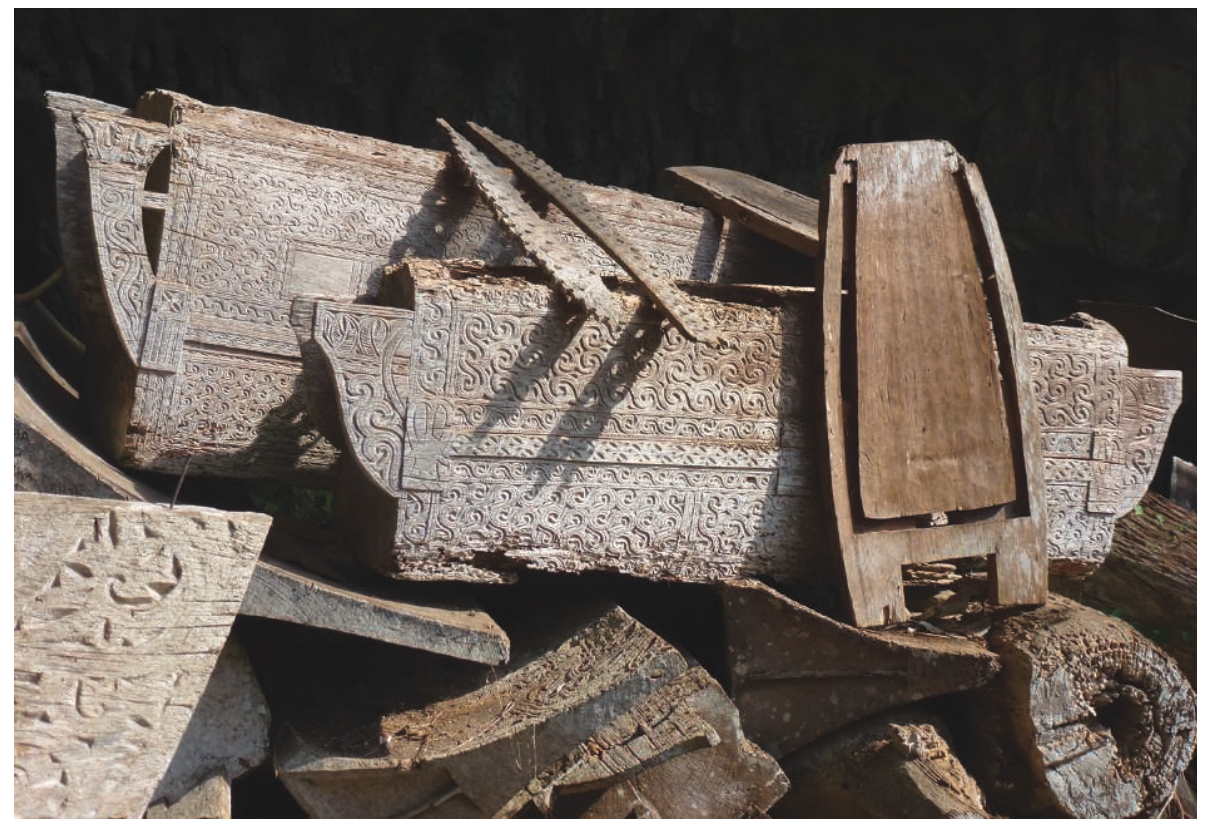

Fig. 12 - D. Rappoport, 2015. Photo Dana Rappoport, novembre 2014.

Légende - « Cercueils en bois (erong), sur le site de sépulture à Lombok Parinding (Canton de Sesean, Toraja Utara). » (p. 200) 


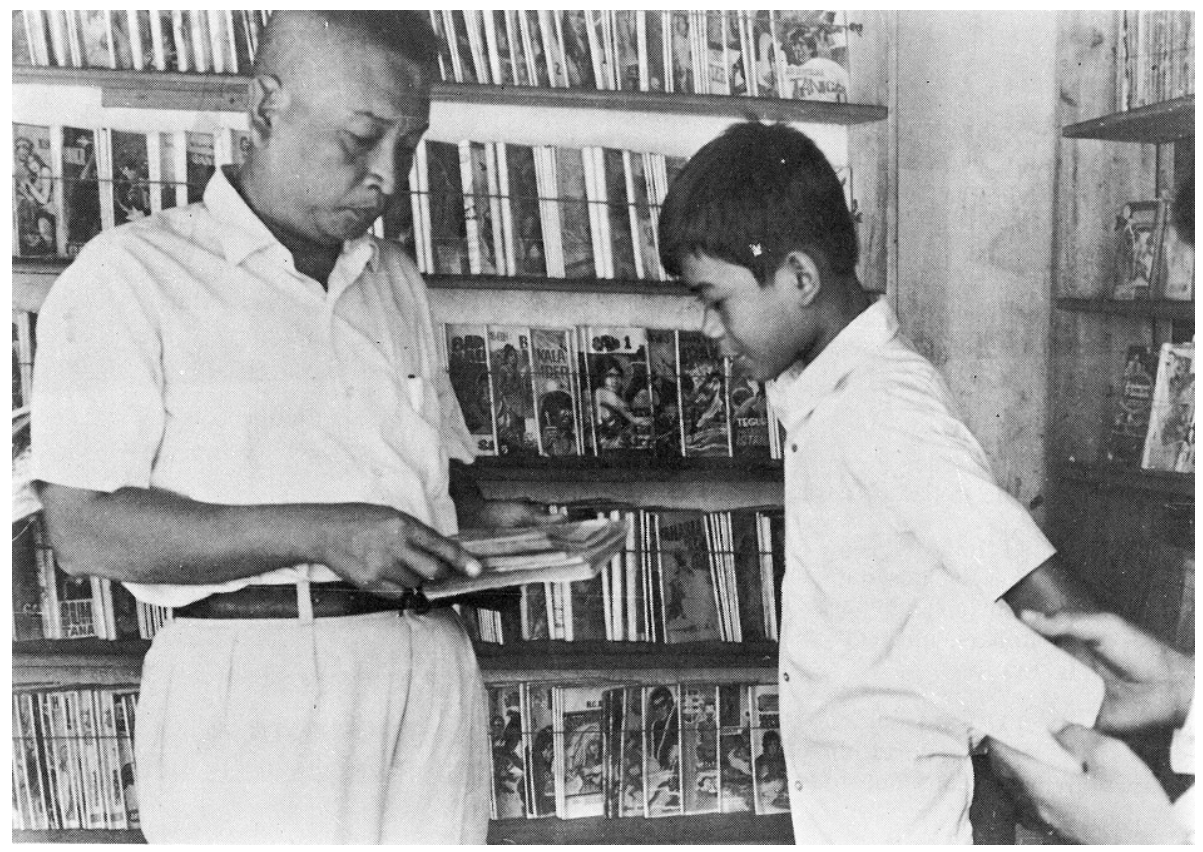

Fig. 13 - Marcel Bonneff, 1972. Photo auteur, s.d.

Légende - « Le choix des ouvrages dans un taman batjaan de Jogjakarta. » (pl. h.t., face p. 192)

Pour finir par où tout aurait pu commencer : jeunesse, lectures, lectures de jeunesse et sujet mineur sur un mode majeur, la bande dessinée en Indonésie.

Qui sont-ils ? Un père et son fils, plutôt que client et vendeur, dans une librairie de bandes dessinées à Yogyakarta, on en devine les couvertures aguicheuses étalées sur les rayonnages. L'autorité paternelle, sérieuse et pincée, est amenée à juger si les ouvrages sollicités par l'enfant lui conviendront ou méritent les quelques dizaines ou centaines de roupies que le vendeur en demandera. Guère de complicité, mais de la componction et une attente pleine d'espoir - tout est dans le dessin des lèvres et l'arc des sourcils. 


\section{Bibliographie}

\section{Références citées dans le texte et les légendes des photos (hors rubriques Images)}

Bonneff M., «Les bandes dessinées en Indonésie : diffusion et public », Archipel, 1972, n 4 , p. $169-178$.

— «Deux images pour un rêve : la bande dessinée et le cinéma indonésiens », Archipel, 1973, $\mathrm{n}^{\circ} 5$, p. $197-208$.

Bougas W.A.L., « Surau Aur: Patani Oldest Mosque », 43, 1992, p. 99-112.

Francino A., « Notes sur les Îles Natuna », 1990, n³9, p. 47-63.

Hamonic G., « Un corps oublié ? À propos d'un cérémonial bissu présenté à Paris (2006) », 2006, nº72, p. 9-14.

Jones R., « Earl, Logan and "Indonesia" », 1973, n6, p. 93-118

Pelras, Chr., « Guide Archipel II », 1975, n¹0, p. 11-50.

— «En mémoire de Denys Lombard », 1998, n55, p. 7-10.

Rappoport D., « Sulawesi, 20 ans après », 2015, n89, p. 179-204.

Rédaction, [Éditorial], 1971, n¹, p. 3.

Salmon Cl., «La communauté chinoise de Surabaya. Essai d'histoire, des origines à la crise de $1930 », 1997, n^{\circ} 53$, p. $121-206$

- «From Cemeteries to Luxurious Memorial Parks with Special Reference to Malaysia and Indonesia », 2016, n 92 , p. 177-212

\section{Les sujets mineurs : " Guides Archipel " et " Pages "}

An., « Guide Archipel (I) : la province de Sumatra Ouest », 1972, n4, p. 115-127.

An., « Pages d'exotisme. 4 : exotisme et cuisine », 1972, n4, p. 128-130.

Bonneff M. \& Voisset G., « Guide Archipel IV : l’île de Sumba », 1980, n¹9, p. 119-141.

Chambert-Loir H., Lombard D. \& Moriceau J., « Guide Archipel V. Bengkulu et son arrièrepays », 1983, n²6, p. 25-42.

Chaillet-Bert J., éd. Archipel, « Pages d'exotisme II : la société javanaise selon J. Chailley-Bert (1900)», 1971, n², p. 107-108.

Labrousse P., « Pages d'exotisme (VII) : La Java des polars », 1974, nº 8, p. 83-88.

_ «Pages d'exotisme (VIII) : Perles dans la rizière », 1976, n¹1, p. 107-112.

_ «Pages d'exotisme (X) : entre les anthropophages et les bolchevistes», 1983, n²5, p. 30-34.

— «Ternate et Tidore. Notes de voyage », 1990, n³9, p. 35-46.

Lombard D., « Pages d'exotisme I : L'Odeur d'llang-ilang de René Ghil », 1971, n¹, p. 103-104.

— «Pages d'exotisme III : Le planteur de Java (1860) de Henri Guénot», 1972, n³, p. 106-112.

_ «Pages d'exotisme (V) : “Un roman d'amour à Java” », 1973, nº, p. 87-90.

— «Pages d'exotisme (VI) : Une utopie en Insulinde : les Lettres de Malaisie de Paul Adam (1898) », 1974, nº , p. 115-118.

— « Pages d'exotisme IX : “Agon, Sultan de Bantan”, Tragédie en 5 actes et en vers », 1978, $\mathrm{n}^{\circ} 15$, p. 53-64.

— « Guide Archipel IV : Pontianak et son arrière-pays », 1984, n²8, p. 77-97.

Lombard-Salmon Cl., Lombard D. \& Labrousse P., " Guide "Archipel” III : Palembang et Bangka, 1978, n 15 , p. 145-161. 
Pelras Chr., « Guide Archipel I : la province de Célèbes-Sud », 1975, n¹0, p. 11-50.

Sellato B., [Pages d'exotisme] «L'“Aventure Vécue” à Bornéo : survol de la "Littérature" ", 1987, n$^{\circ} 11$, p. 143-149.

\section{Les rubriques " Images " $(1991 \rightarrow)$}

Bonneff M. \& Grant St., «"Bons baisers de Batavia”, Cartes postales des Indes Néerlandaises », 1994, n 47 , p. 53-85.

Bonnithon H., « Naissance d'un masque balinais : Hanuman », 1994, n48, p. 49-61.

J.-M. de Grave, « Une école de pencak silat prise sur le vif», 1996, n52, p. 77-93.

Guillot Cl. \& Chambert-Loir H., «Pèlerinage sur la tombe de Sunan Muria (Colo, Java central) », 1993, n45, p. 97-110.

Lombard D., « Le Kampong javanais à l'Exposition Universelle de Paris en 1889 », 1992, $\mathrm{n}^{\circ} 43$, p. $115-130$.

- «À la rencontre d'une société défunte : le cimetière chrétien de Peneleh, à Surabaya ", 1992, n44, p. 123-140.

- [Images et chuchotements] « Des images pour un développement : les panneaux de la Place Merdeka (17 août 1983) », 1993, n46, p. 41-58.

Labrousse P., « Les bouleversements de 1965-1966 dans la documentation de la Fondation Idayu », 1991, n²42, p. 121-131.

— «Images de Timor en France (1812-1824)», 2001, n62, p. 71-90.

— «Auguste de Molins. Un artiste à Java dans Le Tour du monde (1864)», 2009, n78, p. 135-151.

— « Dumont d'Urville et l'Astrolabe à Ambon (1827-1828). Le traitement des images de l'expédition », 2012, n84, p. 175-196.

Laffan M., «Between Batavia and Mecca. Images of Agoes Salim from the Leiden University Library », 2003, n 65 , p. 109-122.

Maurer J.-L., « La gloire du père : images de Suharto à son apogée. Les panneaux du $50^{\mathrm{e}}$ anniversaire de l'indépendance à Jakarta », 1999, n57, p. 73-95.

Njoto H., «À propos d'une pièce en bois sculptée de l'art du Pasisir (XV' ${ }^{\mathrm{e}} . \mathrm{XVII}$ e $\mathrm{s}$. ) : le "kalpataru" du musée de Tuban à Java-Est », 2014, nº8, p. 169-188.

Perret D., «Graha Maria Annai Velangkanni : une église d'inspiration indienne à Medan, Sumatra Nord », 2011, n82, p. 115-136.

Picard M., « Le gambuh : grandeur, décadence et renaissance (?) du théâtre à Bali », 1998, n55, p. 141-190.

Piollet P., Salmon Cl. \& Lombard D., « Une culture révolue ? Le Pasisir javanais de Gresik à Rembang », 1996, n51, p. 95-112.

Salmon Cl., « Les dessins industriels philippins d'Antonio D. Malantie. Une commande de la mission de Lagrené en Chine (1843-1846)», 2004, n67, p. 57-76.

Samuel J., « À la recherche des ateliers perdus. Peinture sous verre et production en série à Java », 2017, n94, p. 143-169.

Siegel J., « Some Views of East Javanese Sorcery », 2002, n64, p. 163-180.

Somers Heidhues M., « An Early Traveler's Compendium: Caspar Schmalkalden's Images of Asia », 2005, nº 70, p. 145-184.

Teh Gallop A., « An Acehnese Style of Manuscript Illumination », 2004, n68, p. 193-240. 